\title{
The effects of hypoxia on the ERG in paediatric cerebral malaria
}

J Lochhead' ', A Movaffaghy², B Falsini³ , S Harding ${ }^{4}$, C Riva ${ }^{2}$ and M Molyneux ${ }^{5}$

\begin{abstract}
Background/aims Cerebral malaria (CM) is a disease of high mortality worldwide. It can be associated with malarial retinopathy (MR) resulting from impaired perfusion within the retinal microvasculature. Areas of capillary nonperfusion (CNP) appear white (retinal whitening) on ophthalmoloscopy. In this study, electrophysiological investigations were performed to investigate the physiological consequences of these hypoxic and ischaemic changes.
\end{abstract}

Methods Children admitted with CM were assessed for inclusion in the study. Those with MR underwent further detailed fundus assessment to quantify retinal whitening and were then designated a severity score. Electrophysiological recordings were performed using a miniganzfeldt stimulator with calibration to the International Society for Clinical Electrophysiology of Visual (ISCEV) standards. ERG data were then analysed with respect to presence of MR and also graded disease severity.

Results Thirty-one children were recruited with a diagnosis of CM, 20 had MR (group 1), and 11 had absent MR (group 2). Statistical analyses of these two groups showed a significant relationship between reduced single flash cone $b$ wave amplitude (CBWA) and increased severity of retinal whitening/ CNP $(P<0.05)$. Cone and maximal response b : a wave ratios remained $>1$ in all subjects. Conclusion Retinal whitening/CNP in MR is associated with significant changes in ERG cone $b$ wave function. The relatively high $b: a$ ratio is compatible with the high frequency of MR resolution without sequelae.

Eye (2010) 24, 259-264; doi:10.1038/eye.2009.162; published online 26 June 2009

Keywords: cerebral; malaria; retinopathy; electrophysiology; ERG
Introduction

Cerebral malaria (CM) is a disease of high morbidity and mortality in malarial endemic areas worldwide. It presents primarily within a paediatric population and is caused exclusively by the Plasmodium falciparum parasite. The disease typically presents with coma of rapid onset, and often with associated convulsions. Other common features include anaemia, hypoglycaemia, and metabolic acidosis.

Several earlier studies have focused on the unique cluster of retinal signs that commonly present within this group of children. Retinal haemorrhages, the majority of which are white centred, retinal whitening (described earlier as cotton wool spots, ${ }^{1,2}$ retinal oedema, ${ }^{1}$ opacification, ${ }^{3}$ or exudation ${ }^{4}$ ), vascular changes mainly within the arterial and capillary bed (described earlier as sheathing or occlusion ${ }^{1-3}$ ), and optic disc swelling. At present the pathophysiology of CM and the associated retinopathy remain unclear. Studies using fluorescein angiography (FFA) have indicated the preservation of an intact blood retinal barrier. ${ }^{3}$ More recent fluorescein angiography studies have confirmed capillary non-perfusion, suggesting hypoxia and ischaemia as the principle underlying pathophysiology. ${ }^{5}$

This study aimed to investigate the effects of the hypoxia and ischaemic retinopathy/ capillary non-perfusion (CNP) (most particularly that of retinal whitening) on the ERG in children with CM retinopathy.

\section{Materials and methods}

For a 3-month period all children admitted to the Malaria Project paediatric high dependency unit at the Queen Elizabeth Central Hospital in Blantyre, Malawi were assessed for inclusion in the study. CM was defined as an inability to localise pain (sternal pressure) in the presence of asexual $P$. falciparum parasitaemia but not
${ }^{1}$ Eye Department, St Mary's Hospital, Newport, Isle of Wight, UK

${ }^{2}$ Institute de Recherche en Ophlatmologie, Lausanne, Switzerland

${ }^{3}$ Istituto di Oftalmologia, Universita Cattolica del S. Cuore, Rome, Italy

${ }^{4}$ St Paul's Eye Unit, Royal Liverpool University Hospital, Liverpool, UK

${ }^{5}$ Malaria Project, Queen Elizabeth Hospital, Blantyre, Malawi

Correspondence:

J Lochhead,

Consultant

Ophthalmologist,

Eye Department,

St Mary's Hospital,

Parkhurst Road,

Newport,

Isle of Wight, PO305 TG, UK

Tel: + 4402380795028 ;

Fax: + 4401983534415

E-mail: jonathan.lochhead@ iow.nhs.uk

Received: 19 March 2009 Accepted in revised form: 31 May 2009 Published online: 26 June 2009 
explained by hypoglycaemia, convulsions, sedatives, or other encephalopathies. All urgent medical interventions including correction of severe hypoglycaemia, anaemia, and commencement of quinine infusion were performed on admission, before commencement of ERG investigation. Ethical committee approval was granted by the Health Sciences Research Committee, and permission for the study was granted by the University of Blantyre College of Medicine.

Children underwent indirect ophthalmoscopy after dilatation with tropicamide $1.0 \%$ and phenylephrine $2.5 \%$. Ophthalmoscopic findings were recorded using standardised charts developed earlier by us and validated in a sample of patients examined by independent, masked observers. For the purposes of this study, the retinal feature under investigation was 'retinal whitening' (Figure 1), which has been shown to represent retinal hypoxia. ${ }^{5}$ Peripheral retinal whitening (PW) was scored on an Ordinal Severity Scale $(0,1+, 2+, 3+)$ for each quadrant and summed to give a composite score. The quadrants were superior, inferior, nasal, and temporal. Macular whitening (MW) was scored by area of involvement $(0,<1 / 3$ disc area (DA), $1 / 3-1 \mathrm{DA}, \geqslant 1 \mathrm{DA})$ within a circle centred on the foveola with a radius of approximately 2.5 disc diameters (the distance from the foveola to the temporal edge of the disc). Within this zone of MW, involvement of the central fovea, referred to as foveal whitening (FW), was scored by quantifying the area

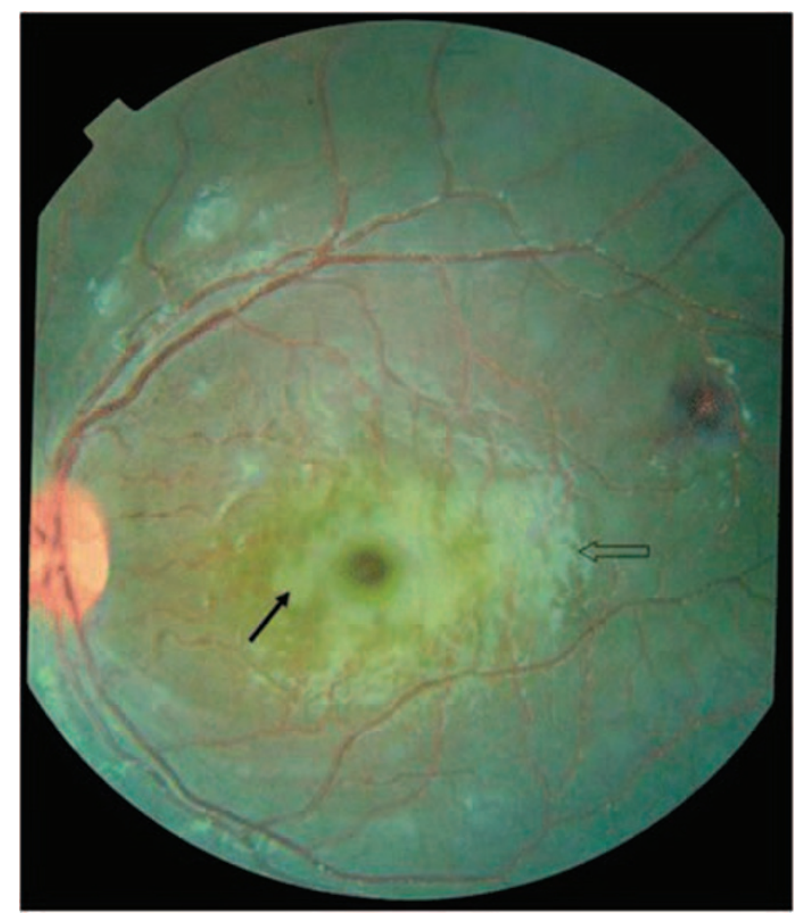

Figure 1 Fundus photograph illustrating foveal whitening (FW) (solid arrow) and surrounding macular whitening (MW). The line arrow represents a camera light reflex. of involvement $(0,<1 / 3,1 / 3-2 / 3, \geqslant 2 / 3)$ within a circle of $500 \mu$ radius centred on the centre of the foveola and roughly equivalent to the zone of retina containing the highest density of cone photoreceptors. A total severity score was then derived by adding together PW, MW, and FW.

ERG recordings were performed using a purpose built compact system, which was versatile and easily transported overseas. The software was installed on a Pentium computer (LACE Elletronica, Pisa, Italy). Hardware comprised a miniganzfeldt stimulator and ADD amplification system with calibration to the International Society for Clinical Electrophysiology of Vision (ISCEV) standards.

Initially, 15 min of dark adaptation was performed by patching of the left eye. The children were then transferred to an appropriately prepared dark room. The previously dilated left eye was then anaesthetised using $0.5 \%$ benoxinate. Children were positioned prone on the right side with the eye to be studied in the plane of the ganzfeldt bowl and dark adapted for a further $5 \mathrm{~min}$ after insertion of gold corneal electrode (JET, Lausanne, Switzerland).

A protocol was performed with the following stimuli: a standard flash calibrated to $2 \mathrm{~cd} / \mathrm{m}^{2} / \mathrm{s}$, a scotopic $\mathrm{dim}$ flash (rod response) calibrated at $2.5 \mathrm{log}$ units of attenuated standard flash, a maximal response using the std. flash, a photopic (cone) response using the std. flash, and following $5 \mathrm{~min}$ of light adaptation at $20 \mathrm{~cd} / \mathrm{m}^{2}$. The $30 \mathrm{~Hz}$ flicker ERG was then recorded. ERG recordings were referenced to a red spot skin electrode at the temple and grounded to a similar electrode at the glabella. A sample of these recordings is illustrated in Figure $2 \mathrm{a}$ and $\mathrm{b}$.

Recordings were stored and analysed using Primus Electrophysiology software (LACE Elletronica). Data from recordings were extracted by two observers (JL, AM) using a consensus protocol with sample validation by a third independent masked observer (BF). Missing or poor data points were excluded from further analysis.

Close scrutiny of all administered quinine was included in our data collection. Time from commencement of most recent quinine infusion was recorded (or'quinine elapsed time'(QET)) along with total cumulative dose and route of administration.

Statistical analysis comparing groups 1 and 2 was performed with $t$ test and analysis of variation (ANOVA). Multiple regression was used to examine associations between the presence of all categories of retinal whitening (PW, MW, and FW), age, and QET with ERG parameters. Linear regression analysis was also used to analyse the relationship between different ERG readings and total severity scores.

We certify that all applicable institutional and governmental regulations concerning the ethical use of human volunteers were followed during this research. 
a
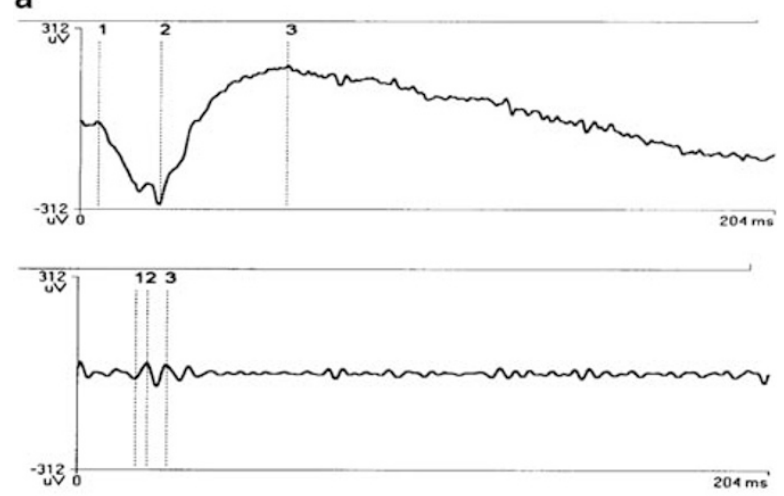

b
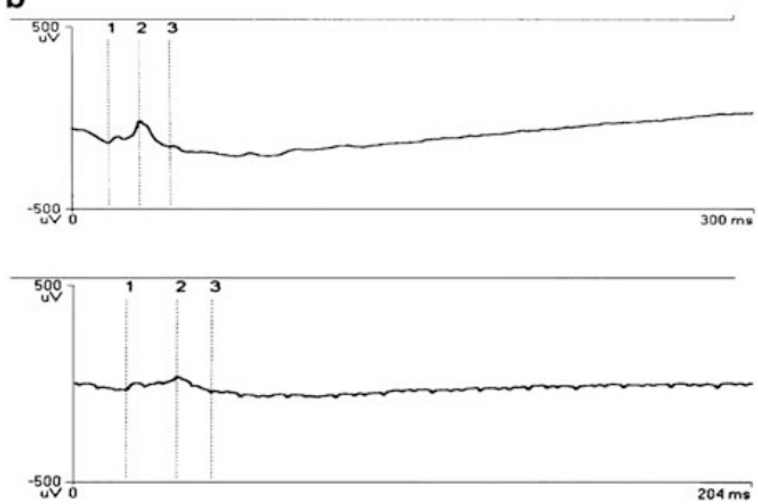

Figure 2 (a) Maximal response recording and derived oscillatory potentials in a 4-year-old subject with mild retinopathy. (b) Single flash photopic ERG in 2-year age-matched subjects with mild (above) and severe retinopathy (below).

\section{Results}

A total of 20 subjects with a diagnosis of CM had 'retinal whitening' visible on fundoscopy and a complete data set for ERG examinations (group1) (Table 1). Only 11 subjects were classified as CM with no 'retinal whitening' (group 2) (Table 2).

Multiple regression analysis of these combined groups was initially performed taking account of age and QET. $\mathrm{CM}$ patients with and without retinopathy provided the following significant associations between single flash cone $\mathrm{b}$ wave amplitude (CBWA) and the individual categories of retinal whitening (MW, FW, and PW), which were graded:

(1) CBWA (dependent variable)/MW (predictor variable): $P<0.05, F=3.32, R^{2}=0.32$. (Variables entered: $\mathrm{MW}(P=0.02)$; age $(P=0.1)$; QET $(P=0.4)$.)

(2) CBWA (dependent variable)/foveal whitening (FW) (predictor variable): $P<0.05, F=3.4, R^{2}=0.24$. (Variables entered: FW $(P=0.031)$; age $(P=0.06)$; QET not significant.)

(3) CBWA (dependent variable)/PW (predictor variable): $P=0.06, F=2.8, R^{2}=0.29$. (Variables entered: $P W$ $(P=0.032)$; age $(P=0.099)$; $\mathrm{QET}(P=0.14)$.)
Table 1 Group 1 demographics, clinical data, and ERG cone b wave amplitude (CBWA)

\begin{tabular}{lccccc}
\hline $\begin{array}{l}\text { Age } \\
\text { (mths) }\end{array}$ & $\begin{array}{c}\text { Central macula } \\
\text { whitening }\end{array}$ & $\begin{array}{c}\text { Central foveal } \\
\text { whitening }\end{array}$ & $\begin{array}{c}\text { Peripheral } \\
\text { whitening }\end{array}$ & $\begin{array}{c}\text { Severity } \\
\text { score }\end{array}$ & $(\mu V)$ \\
\hline 14 & 0 & 0 & 0.50 & 0.50 & 86.1 \\
31 & 1 & 2 & 0.25 & 3.25 & 88.1 \\
61 & 0 & 0 & 0.50 & 0.50 & 95.8 \\
10 & 2 & 2 & 2.00 & 6.00 & 76.4 \\
25 & 2 & 2 & 1.50 & 5.50 & 74 \\
50 & 1 & 1 & 1.75 & 3.75 & 85.2 \\
12 & 2 & 2 & 1.75 & 5.75 & 43.6 \\
31 & 1 & 1 & 1.50 & 3.50 & 71 \\
67 & 1 & 1 & 1.00 & 3.00 & 79.3 \\
25 & 3 & 3 & 2.50 & 8.50 & 65.7 \\
28 & 1 & 1 & 0.00 & 2.00 & 109.4 \\
24 & 3 & 3 & 2.00 & 8.00 & 11.2 \\
32 & 1 & 2 & 0.25 & 3.25 & 81.2 \\
81 & 1 & 0 & 0.00 & 1.00 & 77.5 \\
48 & 1 & 1 & 1.00 & 3.00 & 32.5 \\
38 & 0 & 0 & 1.00 & 1.00 & 77.4 \\
26 & 2 & 1 & 1.50 & 4.50 & 66.3 \\
42 & 3 & 3 & 0.75 & 6.75 & 43.1 \\
10 & 2 & 2 & 2.50 & 6.50 & 81.9 \\
54 & 3 & 3 & 2.50 & 8.50 & 89.1 \\
\hline
\end{tabular}

Table 2 Group 2 demographics, clinical data, and ERG data cone $\mathrm{b}$ wave amplitude (CBWA)

\begin{tabular}{lcc}
\hline Age (mths) & Severity score & $C B W A$ \\
\hline 84 & 0.00 & 57.9 \\
25 & 0.00 & 113.5 \\
10 & 0.00 & 90.5 \\
37 & 0.00 & 95 \\
7 & 0.00 & 92.8 \\
12 & 0.00 & 109 \\
109 & 0.00 & 54.9 \\
72 & 0.00 & 101.1 \\
12 & 0.00 & 62.9 \\
74 & 0.00 & 64.6 \\
10 & 0.00 & 68.1 \\
\hline
\end{tabular}

In summary, CBWA was found to be significantly associated with FW $(P<0.05)$ and MW $(P<0.05)$. The relationship between CBWA and PW approached significance $(P=0.06)$.

Linear regression analysis of severity scores in group 1 vs ERG parameters also showed a relationship between disease severity and CBWA $(P<0.05)$ (Figure 3$)$. In addition within group 1 , there was a significant increase in $30 \mathrm{~Hz}$ flicker implicit time $(P<0.05)$ associated with MW using non-parametric Spearman's rank order analysis.

Cone and maximal response $b$ : a wave ratios remained $>1$ in all subjects (Table 3 ).

Quinine was administered intravenously with a dose calculated from body weight $(\mathrm{kg})$. Elapsed time from most 


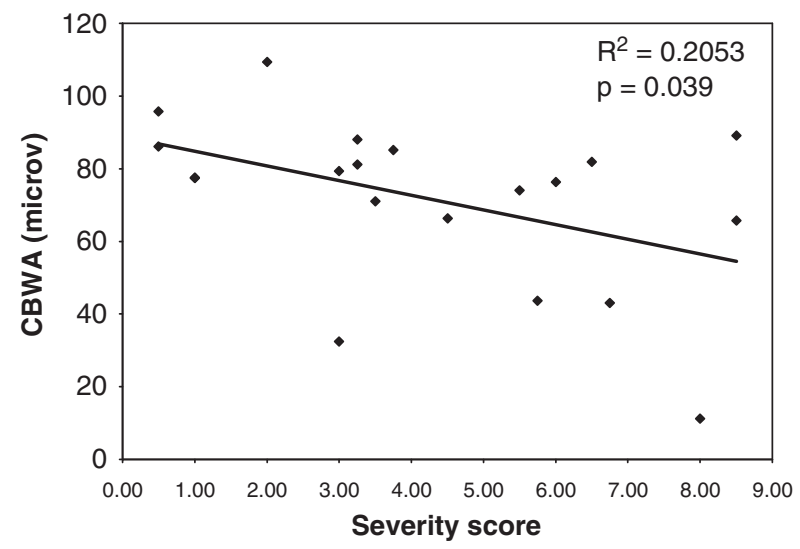

Figure 3 Linear regression scatter plot of severity score vs cone b wave amplitude $(\mu \mathrm{V})$.

Table 3 Comparison of age, cone $\mathrm{b}$ : a ratio, and mean single flash cone $\mathrm{b}$ wave amplitude (CBWA) between groups 1 and 2

\begin{tabular}{lccc}
\hline & $\begin{array}{c}\text { Group 1 } \\
(\mathrm{n}=20)\end{array}$ & $\begin{array}{c}\text { Group 2 } \\
(\mathrm{n}=11)\end{array}$ & P-value \\
\hline Age mean (mths) & 34 & 41 & 0.5 \\
Age Std & 20 & 37 & \\
Cone b: a ratio, mean & 2.91 & 2.57 & 0.23 \\
Cone b: a Std & 0.86 & 1.05 & \\
CBWA mean $(\mu \mathrm{V})$ & 82.8 & 71.4 & 0.2 \\
CBWA Std & 23.1 & 21.5 & \\
& & & \\
& Group 3 & Group 4 & \\
Age mean (mths) & $(n=16)$ & $(n=15)$ & \\
Age Std & 31 & 43 & 0.1 \\
CBWA mean $(\mu \mathrm{V})$ & 34 & 17 & \\
CBWA Std & 68.63 & 83.14 & 0.077 \\
\hline
\end{tabular}

Comparison of age and mean cone $\mathrm{b}$ wave amplitude between groups 3 and 4 .

recently administered quinine infusion (QET) was used to investigate any possible influence on ERG recordings.

The data were initially screened for significant associations using a correlation matrix. A significant positive association was found between QET and both maximal-response a wave amplitude $(P<0.05)$ and cone a wave amplitude $(P<0.05)$. QET and age were not found to influence CBWA.

The mean single flash CBWA was higher in group 2 $(82.8 \mu \mathrm{V})$ than group $1(71.4 \mu \mathrm{V})$; however, this difference did not achieve significance (Table 3).

No significant differences were found between groups 1 and 2 for any other ERG parameter including maximal response $a$ and $b$ waves, cone a wave, maximal response and cone $\mathrm{b}: \mathrm{a}$ wave ratios, oscillatory potentials, and $30 \mathrm{~Hz}$ flicker amplitude. Scotopic rod response was formally excluded because of shortened dark adaptation.

For further analysis the groups were redefined according to disease severity. Sixteen cases had significant retinal involvement (group 3, Table 3) and 15 had absent or minimal retinal involvement (severity score $\leqslant 1$ )(group 4 , Table 3 ).

Statistical data analysis tested the different ERG parameters between these two groups. Mean CBWA in group 3 was $68.63 \mu \mathrm{V}$ compared with $83.14 \mu \mathrm{V}$ in group 4 . Difference in CBWA mean was found to approach significance using ANOVA $(P=0.077)$.

\section{Discussion}

This study comprises the first electrophysiological investigation of retinal whitening or CNP in children suffering from CM retinopathy.

In spite of the difficult field conditions we were able to collect reliable data that are consistent with the expected rise in amplitude of ERG parameters reported by others. ${ }^{6}$ It was not possible to collect data from an age-matched control population. We do have reliable data on children with $\mathrm{CM}$ but with no retinopathy and these may be considered a control group, however the numbers are small (group 2).

There are additional weaknesses in our study. First, the numbers are still relatively small. In a minority of cases circumstances prevented completion of the full period of dark adaptation. A number of patients were restless or hallucinating and this necessitated haste. An inconsistency in the scotopic background environment for these cases has therefore reduced the reliability of our rod response recordings, although the waveforms all seemed appropriate. Eye movements were occasionally present despite coma. These tended to be either random and intermittent or occasionally more consistent with an underlying neurological syndrome, for example Nystagmus or tonic deviation. In many cases the presence of a corneal contact lens electrode helped to stabilise the eye and also to keep the lids open. Any waveforms collected with an unacceptable amount of noise were either repeated or rejected.

We have showed with a variety of statistical methods a relationship between retinal whitening and decreased cone function (Figure 2b). Single flash CBWA is associated with the presence of increased severity of retinal whitening $(P<0.05)$. No significant difference was found, however, in a direct statistical comparison of the means between groups 1 and 2. This may be the result of relatively small numbers in group 2 . It is possible that cases with minimal visible disease according to our grading system still have undetectable 
electrophysiological changes within the retina. If this is the case then subjects with lower severity scores would help to show a significant effect in multiple and linear regression analyses within the affected group but would reduce any detectable differences in comparison with the unaffected controls.

To explore this further the groups were redefined. Sixteen cases had significant retinal involvement (group 3, Table 3) and 15 had absent or minimal retinal involvement (severity score $\leqslant 1$ )(group 4 , Table 3 ).

Statistical data analysis between these two groups did show a difference in mean single flash CBWA in group 3 of $68.63 \mu \mathrm{V}$ compared with $83.14 \mu \mathrm{V}$ in group 4 . In this analysis the difference in CBWA was found to approach significance $(P=0.077)$. This may indicate that lower severity scores on our grading system do not yet have detectable ERG changes.

Flicker IT did seem to be affected in our malarial retinopathy (MR) group but there were no statistically significant changes detected for $30 \mathrm{~Hz}$ Flicker amplitude or oscillatory potentials as one might expect. This may, in part, be due to the small sample sizes.

CNP has been topographically linked to the area of retinal whitening. ${ }^{5}$ These areas are therefore thought to represent hypoxia and ischaemia from sequestration of parasitised red blood cells. Our findings of a positive correlation between retinal whitening and reduced CBWA help to support this theory because the latter is known to be very sensitive to hypoxia. ${ }^{7-9}$

The $b$ : a wave ratio has been shown to be a good prognostic indicator in ischaemia, $<1$ being associated with a poor outcome. ${ }^{8-10}$ The $\mathrm{b}:$ a ratios in our subjects were always $>1$ (Table 3 ). These high ratios are compatible with the good visual outcomes observed without sequelae. ${ }^{8}$ It has also been described elsewhere that observed MW seems to be reversible with no adverse visual sequelae. ${ }^{11}$

Current data obtained earlier from healthy children, show increasing ERG amplitudes with increasing age. ${ }^{6}$ This was therefore identified as an anticipated source of bias before our study. Groups 1 and 2, however, seem to be well age matched (Table 3 ).

Children with CM are treated with high doses of intravenous quinine, which is known to have effects on retinal electrophysiology in overdose. ${ }^{12-15}$ Elapsed time from most recently administered quinine infusion (QET) was measured. A significant positive association was found between QET and both maximal-response a wave amplitude $(P=0.03)$ and cone a wave amplitude $(P=0.04)$. It was concluded that parenteral quinine administered in therapeutic doses to a paediatric population seems to cause a transient depression in photoreceptor function. No evidence of ocular quinine toxicity was found at the therapeutic doses used. These data have been published elsewhere. ${ }^{16}$ QET and age were not found to influence CBWA.

Our findings seem to show increased sensitivity of cone second-order neurones to hypoxia. This is supported elsewhere in the medical literature. ${ }^{7,8,17}$ It is possible that cone second-order neurones with their more complex and demanding functions are more sensitive to any metabolic or physiological insult. Photoreceptors and RPE receive additional circulation from choroidal vascular supply.

Fundus fluorescein angiograms performed on these patients more recently show capillary microvascular occlusion..$^{5}$ Parasitised erythrocytes sequester in the microvascular circulation by cytoadherence through upregulation of red cell surface receptors. The ERG data presented in this study support the theory that retinal whitening is caused by oncotic swelling of second-order neurones in the inner retinal layer because of metabolic or hypoxic stress. ${ }^{3}$ A mechanism of metabolic steal by intravascular sequestered parasites has been suggested. ${ }^{11,18}$

In conclusion these data show that the presence of increasingly severe retinal whitening (retinal CNP/ hypoxia) in CM retinopathy is associated with significant changes in cone b wave function.

\section{Conflict of interest}

The authors declare no conflict of interest.

\section{References}

1 Lewallen S, Taylor TE, Molyneux ME, Wills BA, Courtwright P. Ocular fundus findings in Malawian children with cerebral malaria. Ophthalmology 1992; 100: 857-861.

2 Davis TME, Suputtamongkol Y, Spencer JL, Ford S, Chienkul W, Schulenburg WE et al. Measures of capillary permeability in acute falciparum malaria: relation to severity of infection and treatment. Clin Infect Dis 1992; 15: 256-266.

3 Hero M, Harding SP, Riva C, Winstanley PA, Marsh K. Photographic and angiographic characterisation of retinal appearances in severe malaria in Kenyan children. Arch Ophthalmol 1997; 115(8): 997-1003.

4 Davis MW, Vaterlaws AL, Simes J, Torzillo P. Retinopathy in malaria. PNG Med J 1982; 25: 19-22.

5 Beare NA, Harding SP, Taylor TE, Lewallen S, Molyneux ME. Perfusion abnormalities in children with cerebral malaria and malarial retinopathy. J Infect Dis 2009; 1999(2): 263-271.

6 Birch DG, Anderson JL. Standardised full field electroretinography, normal values and their variation with age. Arch Ophthalmol 1992; 110: 1571-1576.

7 Block F, Schwarz M. The b-wave of the electroretinogram as an index of retinal ischaemia. Gen Pharmacol 1998; 30(3): 281-287.

8 Matsui Y, Katsumi O, Mehta MC, Hirose T. Correlation of electroretinographic and fluorescein angiographic findings 
in unilateral central retinal vein occlusion. Graefes Arch Clin Exp Ophthalmol 1994; 232(8): 449-457.

9 Breton ME, Quinn GE, Keene SS, Dahman JC, Brucker AJ. Electroretinogram parameters at presentation as predictors of rubeosis in central retinal vein occlusion patients. Ophthalmology 1989; 96(9): 1343-1352.

10 Sabates R, Hirose T, McMeel JW. Electroretinography in the prognosis and classification of central retinal vein occlusion. Arch Ophthalmol 1983; 101(2): 232-235.

11 Beare N, Southern C, Kayira K, Taylor T, Harding SP. Visual outcomes in children in Malawi following retinopathy of severe malaria. Br J Ophthalmol 2004; 88(3): 321-324.

12 Buchanan TA, Lyness RW, Collins AD, Gardiner TA, Archer DB. An experimental study of quinine induced blindness. Eye 1987; 1(pt 4): 522-524.

13 Zahn JR, Brinton GF, Norton E. Ocular quinine toxicity followed by electroretinogram, electro-oculogram, and pattern visually evoked potentials. Am J Optom Physiol Opt 1981; 58(6): 492-498.

14 Canning CR, Hague S. Ocular quinine toxicity. $\mathrm{Br}$ J Opthalmol 1988; 72: 23-26.

15 Bacon P, Spalton DJ, Smith SE. Blindness from quinine toxicity. Br J Opthalmol 1988; 72: 219-224.

16 Lochhead J, Movaffaghy A, Falsini B, Winstanley PA, Mberu EK, Riva C et al. The effect of quinine on the electroretinopgrams of children with paediatric cerebral malaria. J Infect Dis 2003; 187(8): 1342-1345.

17 Hayreh SS, Klugman MR, Podhajsky P, Kolder HE. Electroretinography in central retinal vein occlusion. Correlation of electroretinographic changes with pupillary abnormalities. Graefes Arch Clin Exp Ophthalmol 1989; 227(6): 549-561.

18 Beare N, Taylor T, Harding SP, Lewallen S, Molyneux M. Malarial retinopathy: a newly established diagnostic sign in severe malaria. Am J Trop Med Hyg 2006; 75(5): 790-797. 